\title{
CONTRIBUCION A LA FLORA DE MIXOMICETES DE CATALUÑA
}

\author{
por \\ ENRIC GRACIA BARBA
}

Departamento de Botanica. Universidad de Barcelona. Barcelona

\section{INTRODUCCION}

Este trabajo resume el estudio de muestras recogidas en 22 localidades de Cataluña, desde el año 1972 al 1976.

Hemos hallado en la bibliografía citas para Cataluña correspondientes a 21 especies. El catálogo preparado por Torre-Calonge (1975), cita para la totalidad de España 51 especies. Entre las 192 muestras recolectadas, hemos reconocido 48 especies distintas, de las cuales 35 son nuevas para Cataluña, cuyo catálogo pasa a ser de 56 especies, y 27 no estaban incluidas en el catálogo de Torre-Calonge (1975), con lo cual la lista de especies españolas asciende a 78.

En la Fig. 1, se señalan las 22 localidades estudiadas, que corresponden a:

I. Provincia de Gerona.

1. Planolas; 2. Tossa de Mar; 3. Blanes; 4. Hostalric.

II. Provincia de Barcelona.

1. Sta. María de Cervelló; 2. Tibidabo; 3. Montseny; 4. Conrerias; 5. Bruguers; 6 . Collsacreu-Olzinelles; 7. Sta. Eulalia de Ronsana; 8. Gelida; 9. Bellaterra; 10. Prats del Rei; 11. Orrius.

III. Provincia de Tarragona.

1. Delta del Ebro; 2. Tortosa; 3. Sierra de Prades; 4. Poblet.

IV. Provincia de Lérida.

1. Espot; 2. Viella; 3. Tabescán. 


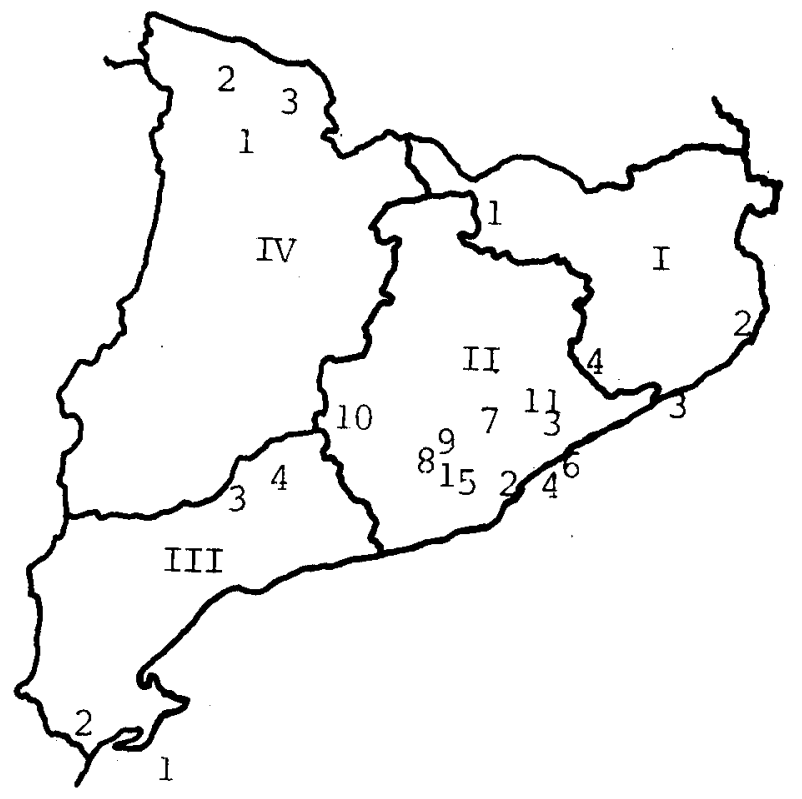

Fig. 1. Mapa de Cataluña. Las provincias se indican por números romanos, las localidades por números anábigos. 
De cada especie señalada en el catálogo indicamos las localidades donde se ha recolectado, el nombre del recolector (EG si es el autor), el número de orden del herbario (EGB/115), fecha de recolección, substrato, si se ha hallado utilizando la cámara húmeda y señalamos por Rev. Nann-Brem. aquellas especies cuya identificación ha sido revisada por N.E. Nannenga-Bremekamp. Así como, señalamos con un asterisco $\left({ }^{*}\right)$, las especies nuevas para Cataluña, y con dos (**), las especies nuevas para España.

La clasificación adoptada es la de Alexopoulos (1973). Los nombres de las especies están de acuerdo con la taxonomía de Martin-Alexopoulos (1969). No obstante se han consultado paralelamente en los casos críticos las obras de Nannenga-Bremekamp (1976) y Lister (1925).

\section{RESULTADOS. LISTA DE ESPECIES}

Ceratiomixáceas.

* Ceratiomyxa fruticolosa (Mull.) Macbr.

Planoles, EG, EGB/22, VIII. 1973, sobre Pinus sylvestris; Planoles, EG, EGB/77, 3. VIII. 1974, sobre Pinus mugo ssp. uncinata; Planoles, EG, EGB/176, 16.VII.1976, sobre Pinus sylvestris; Tortosa, Bertault, EGB/151, 29.X.1974, sobre Pinus sylvestris; Viella, Girbal, EGB/145, 25.VIII.1975, sobre Pinus sp.

\section{Liceáceas.}

\section{* Licea minima Fries.}

Planoles, EG, EGB/28, VIII.1973, sobre Pinus sylvestris, Rev. Nann. - Brem.

* Lícea variabilis Schrader.

Cervelló, A. Carrillo, EGB/159, 2.IV.1976, sobre Populus nigra en cámara húmeda.

Reticulariáceas.

Tubifera ferruginosa (Batsch) Gmel.

Blanes, EG, EGB/9, IX.1972 Pinus halepensis.

* Dictydiaethalium plumbeum (Schum.) Rost.

Tibidabo, Girbal, EGB/106, 25.III.1975, sobre Corylus avellana; 
Planoles, X. de los Santos, EGB/163, 1.l.1976, sobre Betula sp.Rev. Nann.-Brem.

Lycogala epidendrum (L.) Fries.

Planoles, EG, EGB/30, VII.1973, sobre Pinus sylvestris; Planoles, EG, EGB/10, 10.IX.1972, sobre Pinus sylvestris; Montseny, X. Llimona, EGB/130, 20.X.1974, sobre Picea excelsa; Conrerias, EG, EGB/95, 19.X.1974, sobre Pinus halepensis; Espot, EG, EGB/59, 18.VII.1974, sobre Pinus mugo ssp uncinata.

Reticularia lycoperdon Bull.

Blanes, EG, EGB/11, 24.IX.1972, sobre Pinus halepensis; Tossa de Mar, X. Llimona, EGB/129, 18.V.1974, sobre Pinus halepensis.

* Reticularia jurama Meylan.

Planoles, EG, EGB/62, 18.VII.1974, sobre Pinus mugo ssp uncinata.

Cribrariáceas.

* Lindbladia tubulina Fries.

Cervelló, X. Llimona, EGB/135, 29.X.1975, substrato no identificado. Rev. Nann.-Brem.; Cervelló, EG, EGB/4, VIII.1972, sobre musgo.

Cribraria aurantiaca Schrad.

Planoles, EG, EGB/82, 18. VIII.1974, sobre Pinus sylvestris.

Cribraria argillacea (Pers.) Pers.

Planoles, EG, EGB/75, 3.VIII.1S74, sobre Pinus sylvestris; Planoles, EG, EGB/76, 3.VIII.1974, sobre Pinus sylvestris; Planoles, EG, EGB/83, 2.IX.1974, sobre Pinus sylvestris.

* Cribraria violacea Rex.

Prat del Rei, EG, EGB/165, 7.V.1976, sobre Pinus nigra.

* Dictydium cancellatum (Batsch.) Macbr.

Planoles, EG, EGB/19, VIII.1973, sobre Pinus sylvestris; Cervelló, A. Rosell, EGB/37, 7.X.1973, sobre Pinus halepensis; Conrerias, EG, EGB/96, 19.X.1974, sobre Pinus halepensis; Tabescán, Girbal, $\mathrm{EGB} / 142$, 23. VIII.1975, sobre Abies alba.

Equinosteliáceas. 
* Echinostelium minutum de Bary.

Prades, EG, EGB/187, 8.XI.1976, sobre Castanea sativa en cámara húmeda.

Dianemáceas.

* Dianema corticatum A. Lister.

Delta del Ebro, EG, EGB/157, 7.III.1976, sobre Populus sp en cámara húmeda. Rev. Nann-Brem.

Triquiáceas.

* Perichaena chrysosperma (Currey). A. Lister.

Blanes, EG, EGB/12, 8.XI.1972, sobre Robinia pseudoacacia.

* Perichaena vermicularis (Schw.) Rost.

Delta del Ebro, EG, EGB/156, 7.III.1976, sobre Populus alba en cámara húmeda.

* Perichaena corticalis (Batsch.) Rost.

Planoles, EG, EGB/8, 5.IX.1972, sobre madera no identificada.

Arcyria ferruginea Sauter.

Bruguers, X. Llimona, EGB/126, III.1974, sobre Pinus halepensis.

Arcyria nutans (Bull.) Grev.

Tibidabo, EG, EGB/99, 21.X.1974, sobre madera no identificada. Rev. Nann-Brem.; Montseny, X. Llimona, EGB/133, 6.IV.1974, sobre Quercus pubescens.

* Arcyria pomiformis (Leers) Rost.

Tibidabo, EG, EGB/99, 21.X.1974, sobre Quercus cerrioides. Rev. Nann.-Brem.

* Arcyria cinerea (Bull.) Pers.

Planoles, EG, EGB/18, VIII.1973, sobre Betula sp.; Planoles, EG, EGB/79, 25.VIII.1974, sobre Pinus sylvestris; Tibidabo, X. Llimona, EGB/105, 8.IV.1975, sobre Quercus cerrioides. Rev. Nann,-Brem.

* Arcyria insignis Kalchbr. \& Cooke.

Cervelló, X. Llimona, EGB/79, VIII.1974, sobre Pinus halepensis; Mont- eny, A. Carrillo, EGB/98, 20.X.1974, sobre substrato no identifi- 
cado; Tibidabo, J. Girbal, EGB/101, 1.XI.1974, sobre Quercus cerrioides.

* Prototrichia metallica Mass.

Espot, EG, EGB/64, 20.VII.1974, sobre Pinus sylvestris.

Trichia varia (Pers.) Pers.

Montseny, X. Llimona, EGB/102, XI.1974, sobre Fagus sylvatica; Collsacreu, A. Carrillo, EGB/104, XI.1974, sobre madera desconocida; Olzinelles, A. Tomás, EGB/100, 31.X.1974, sobre Quercus suber; Olzinelles, X. Llimona, EGB/153, 31.X.1974, sobre Quercus suber.

Trichia favoginea (Batsch.) Pers.

Olzinelles, X. Llimona, EGB/149, sobre Quercus suber; Olzinelles, X. Llimona, EGB/148, sobre Quercus suber.

Fisaráceas.

Leocarpus fragilis (Dicks.) Rost.

Orrius, Sr. Boix, EGB/132, 8.IV.1974, sobre Pinus pinea; Planoles, EG, EGB/127, 2.IV.1975, sobre Galium sp. vivo.

* Badhamia foliicola A. Lister.

Blanes, EG, EGB/13, 8.XII.1972, sobre madera desconocida.

* Badhamia affinis Rost.

Blanes, EG, EGB/2, V.1972 sobre Cupressus sp.

* Badhamia panicea (Fries) Rost.

Cervelló, A. Carrillo, EGB/155, 2.IV.1976, sobre Populus nigra. Rev. Nann.-Brem.

Fuligo septica (L.) Wiggers.

Planoles, EG, EGB/29, VIII.1973, sobre Juniperus communis; Planoles, EG, EGB/84, 26.VIII.1974, sobre muro de piedra; Gelida, X. Llimona, EGB/141, 1.V.1975, sobre Populus deltoides. Rev. Nann.Brem.

* Physarum nutans Pers.

Prades, EG, EGB/138, 19.IV.1975, sobre Querus cerrioides. Rev. Nann.-Brem. 
* Physarum viride (Bull.) Pers.

Planoles, EG, EGB/73, 3.VIII.1974, sobre Pinus sy/vestris.

* Physarum compressum Alb. \& Schw.

Blanes, EG, EGB/3, VI.1972, sobre Robinia pseudoacacia.

* * Physarum vernum Fries. tris.

Montseny, X. Llimona, EGB/34, 23.X.1973, sobre Pinus sylves-

* Physarum decipiens Curtis.

Montseny, X. Llimona, EGB/170, 8.IX.1976, sobre Quercus pubescens.

* Physarum virescens Ditmar.

Planoles, EG, EGB/177, 16. VII.1976, sobre Scabiosa sp. viva.

* Craterium leucocephalum (Pers.) Ditmar.

Prades, EG, EGB/139, 19.IV.1975, sobre llex aquifolium y Hedera helix vivas. Rev. Nann.-Brem.

Didimiáceas.

* Diachea leucopodia (Bull.) Rost.

Hostalric, EG, EGB/132, XI.1974, sobre Populus sp.

Mucilago crustacea Wiggers.

Sta. Eulalia de Ronçana, E. Velasco, EGB/143, 7.IV.1974, sobre Calluna vulgaris y Cistus salvifolius; Aiguafreda, X. Llimona, EGB/178, 18.X.1976, substrato desconocido.

* Diderma haemisphaericum (Bull.) Hornema.

Tibidabo, X. Llimona, EGB/134, 6.V.1974, sobre UImus sp.

* Didymium squamulosum (Alb. \& Schw) Fries.

Bellaterra, EG, EGB/161, 10.IV.1976, sobre Ulmus sp. en cámara húmeda. Rev. Nann.-Brem.

* Didymium dubium Rost.

Poblet, EG, EGB/154, 29.X.1975, sobre llex aquifolium. Rev. Nann.-Brem. 


\section{Estemonitáceas.}

* Enerthenema papillatum (Pers.) Rost.

Planoles, EG, EGB/73, 30.VII.1974, sobre Pinus sylvestris; Espot, EG, EGB/67, 24. VII. 1974, sobre Pinus sy/vestris.

* Enerthenema melanosporum Macbr. \& Martin.

Espot, EG, EGB/69, 24.VII.1974, sobre Pinus sylvestris; Espot, EG, EGB/70, 24.VII.1974, sobre Pinus sylvestris; Espot, EG, EGB/71, 24. VII.1974, sobre Pinus sylvestris. Rev. Nann-Brem.

Stemonitis fusca A. Lister.

Planoles, EG, EGB/81, VIII.1974, sobre Pinus sylvestris; Tibidabo, A. Romo, EGB/131, 15.XII.1974, sobre madera desconocida; Blanes, EG, EGB/1, VIII.1972, sobre madera desconocida.

* Stemonitis axifera (Bull.) Macbr.

Planoles, EG, EGB/6, 16. VIII.1972, sobre Pinus sylvestris.

* Comatricha nigra (Pers.) Schroet.

Tibidabo, J. Girbal, EGB/107, 25.III.1975, sobre Corylus avellana; Planoles, EG, EGB/86, 18.VIII.1974, sobre Pinus sp.; Ripoll, EG, EGB/93, 10.VI.1975, sobre Pinus sp.

* Comatricha elegans (Racib.) G. Lister.

Planoles, EG, EGB/37, VIII.1973, sobre Pinus sylvestris.

\section{RESUMEN}

Se presenta una lista de 48 especies de mixomicetes, de las cuales 35 son nuevas para Cataluña, cuyo catálogo pasa a ser de 56 especies, y 27 no estaban incluidas en el catálogo para España, con lo cual la lista de especies españolas asciende a 78.

Enerthenema melanosporum Macbr. \& Martin, sólo había sido citada en dos ocasiones para Europa.

\section{AGRADECIMIENTOS}

Se agradece en especial la ayuda prestada por el Dr. X. Llimona Pagés en la dirección de este trabajo, a N. E. NannengaBremmekamp en la revisión de las identificaciones, así como a 
personal del Departamento de Botánica, colaboradores de la. Sociedad Catalana de Micología, y amigos, que me han ayudado en la recolección de material.

\section{BIBLIOGRAFIA}

LISTER, A. - 1925. A Monograph of the Mycetozoa. Brit. Mus. N. H. London.

MARTIN, G. W. y ALEXOPOULOS, C. J. - 1969. The Myxomycetes. University of lowa Pres, lowa City.

NANNENGA - BREMEKAMP, N. E. - 1976. De Nederlandse Myxomyceten. Kon. Neder, Natuur. Ver Amsterdam.

TORRE, M. de la, y CALONGE, F. D. - 1975. Contribución al catálogo de los myxomycetes de España. I. Inst. Bot. Cavanilles. 1 (32): 89-99.

ALEXOPOULOS, C. J. - 1973. Myxomycetes. En THE FUNGI, an advanced treatise. (G. C. Ainsworth, F. K. Sparrow, y A. S. Sussman, eds.). Vol. IVB. 3: 39-60. Acad. Press, New York. 